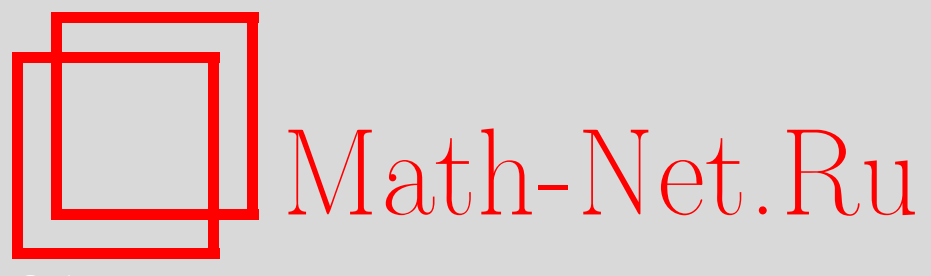

В. П. Гришухин, В. И. Данилов, Г. А. Кошевой, Унимодулярные системы векторов вложимы в $(0,1)$-куб, $M a$ тем. заметки, 2010, том 88, выпуск 6, 938-941

DOI: https://doi.org/10.4213/mzm8918

Использование Общероссийского математического портала Math-Net.Ru подразумевает, что вы прочитали и согласны с пользовательским соглашением http://www.mathnet.ru/rus/agreement

Параметры загрузки:

IP: 54.172 .240 .79

26 апреля 2023 г., 16:34:20

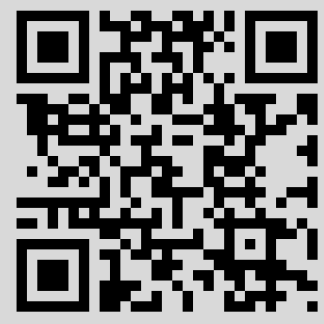




\title{
Унимодулярные системы векторов вложимы в $(0,1)$-куб
}

\author{
В. П. Гришухин, В. И. Данилов, Г. А. Кошевой
}

Введение. Существуют по крайней мере три важных области применения унимодулярных систем: целочисленное программирование, дискретная выпуклость и точечные решетки.

Всякий многогранник, линейные ограничения которого определяются унимодулярной системой, имеет вполне унимодулярную матрицу ограничений. Поэтому такой многогранник целочисленен, т.е. все его вершины целые, и задачу оптимизации линейного функционала на нем можно решать хорошо разработанными методами линейного программирования (см., например, [1]).

Существует большое число работ, посвященных определению и изучению дискретных выпуклых множеств. Но на наш взгляд наиболее плодотворным является определение дискретного выпуклого множества, связанное с унимодулярными системами. В частности, для таких множеств экономики с неделимыми товарами в модели Эрроу-Гурвица имеют равновесие. Такое определение приводит к двум классам $S$ и $I$ дискретных выпуклых множеств. Класс $S$ замкнут относительно суммы Минковского, а класс $I$ замкнут относительно пересечения. Оба класса определяются унимодулярными системами $U$ и состоят из многогранных множеств $P$ таких, что ребра $P$ параллельны векторам из $U$, если $P \in S$, и линейные ограничения $P$ определяются системой $U$, если $P \in I$ (см. [2], [3]).

Имеется специальный класс точечных трансляционных решеток, называемых дайсингами. Эти решетки получаются как точки пересечения бесконечных семейств параллельных эквидистантных гиперплоскостей. Такое пересечение дает решетку тогда и только тогда, когда гиперплоскости семейств ортогональны векторам некоторой унимодулярной системы. Дайсинг решетка замечательна, в частности, тем, что ее многогранник Вороного есть зонотоп, являющийся суммой Минковского отрезков, параллельных векторам унимодулярной системы, определяющей эту решетку (см. [4]).

Многогранник Вороного является частным случаем параллелоэдра, т.е. такого многогранника, параллельные трансляции которого заполняют все пространство без зазоров и пересечений по внутренним точкам. Зонотоп является параллелоэдром тогда и только тогда, когда он есть сумма отрезков, параллельных векторам некоторой унимодулярной системы.

Ниже под словами "унимодулярная система" подразумевается множество векторов, взятых по одному из каждой пары противоположных векторов. Такая система представляет некоторый регулярный матроид (см. [5]).

В исследованиях по гиперметрикам и положительно полуопределенным формам возник вопрос: представляется ли всякая унимодулярная система $(0,1)$-векторами, т.е. характеристическими векторами семейства подмножеств некоторого множества (см. [6]). Хорошо известно, например, что характеристические векторы ламинарного семейства множеств образуют некоторую унимодулярную систему.

В работе [7] показано, что всякая максимальная унимодулярная система представима в виде $k$-суммы, где $k=0,1,2$, графической, кографической и специальной 5 -мерной $\mathbf{E}_{5}$ унимодулярных систем. Максимальная $n$-мерная графическая система представима, в частности, всеми корнями знаменитой системы корней $\mathbf{A}_{n}$. Эта система корней является векторным представлением графического матроида $M\left(K_{n+1}\right)$ полного графа $K_{n+1}$ 
на $n+1$ вершинах. Если в качестве базы $M\left(K_{n+1}\right)$ взять $n$ ребер некоторого гамильтонового пути в $K_{n+1}$, то все векторы унимодулярной системы $\mathbf{A}_{n}$, представляющие ребра $K_{n+1}$, будут иметь $(0,1)$-координаты в базисе, соответствующем этой базе.

Любая максимальная $n$-мерная кографическая унимодулярная система представляет кографический матроид $M^{*}(G)$ трехсвязного кубического графа $G$ на $2(n-1)$ вершинах. Можно показать, что если граф $G$ имеет гамильтонов путь, то матроид $M^{*}(G)$ представим $(0,1)$-векторами. Но, к сожалению, неизвестно, имеет ли всякий трехсвязный кубический граф гамильтонов путь. Из результата этой заметки естественно возникает гипотеза о существовании гамильтонового пути в любом кубическом трехсвязном графе. Следует заметить, что существуют трехсвязные кубические графы, не имеющие гамильтоновых ииклов (см. [8]).

Исключительная унимодулярная система $\mathbf{E}_{5}$ содержит, с точностью до знака, 10 векторов. Она представляет матроид $R_{10}$, играющий важную роль в работе Сеймура [5] о разложении регулярных матроидов в $k$-сумму. Матроид $R_{10}$ есть такое расширение графического матроида $M\left(K_{33}\right)$ двудольного графа $K_{33}$ гиперребром $\Omega$, что $\Omega$ образует цикл мощности 4 с каждым паросочетанием графа $K_{33}$. Легко проверить, что любая из двух матриц, составленная из характерестических векторов либо всех десяти двухэлементных подмножеств, либо всех десяти трехэлементных подмножеств некоторого пятиэлементного множества, представляет унимодулярную систему $\mathbf{E}_{5}$.

Ниже мы даем явное соответствие ребер графа $K_{33}$ и гиперребра $\Omega$ двухэлементным множествам $i j, 1 \leqslant i<j \leqslant 5$. Обозначим множества вершин двух долей графа $K_{33}$ через $\{A B C\}$ и $\{a b c\}$. Пусть $u(X y)$ есть двухэлементное множество, сопоставленное дуге $(X y)$, идущей из вершины $X$ в вершину $y$. Имеем

$$
\begin{aligned}
& u(A a)=12, \quad u(B a)=24, \quad U(C a)=25, \\
& u(A b)=13, \quad u(B b)=34, \quad U(C b)=35, \quad u(\Omega)=23, \\
& u(c A)=45, \quad u(c B)=15, \quad U(c C)=14 \text {. }
\end{aligned}
$$

Всякая унимодулярная система определена с точностью до невырожденного линейного преобразования. Множество таких унимодулярных систем образует класс эквивалентных унимодулярных систем. В этой работе мы показываем, что в любом классе эквивалентности существует унимодулярная система и базис пространства этой системы, в котором ее векторы имеют $(0,1)$-координаты. Стоит подчеркнуть, что этот базис может не принадлежать этой унимодулярной системе.

Основной результат. Пусть $U$ есть $n$-мерная унимодулярная система, векторы которой порождают все пространство $\mathbb{R}^{n}$. Каждый вектор $u \in U$ определяет следующее бесконечное семейство гиперплоскостей:

$$
\mathscr{H}(u)=\left\{H_{k}(u): k \in \mathbb{Z}\right\},
$$

где

$$
H_{k}(u)=\left\{x \in \mathbb{R}^{n}: u^{T} x=k\right\} .
$$

Здесь через $a^{T} b$ обозначено скалярное произведение векторов $a, b \in \mathbb{R}^{n}$.

Пусть $B \subseteq U$ есть произвольное базисное подмножество. Так как $U$ есть унимодулярная система, то каждый вектор $u \in U$ имеет целочисленное разложение по векторам $b \in B: u=\sum_{b \in B} z_{b} b$, где, более того, $z_{b} \in\{0, \pm 1\}$. Очевидно, что пересечение $\bigcap_{b \in B} H_{k_{b}}(b)$ есть точка, являющаяся решением системы уравнений

$$
b^{T} x=k_{b}, \quad \text { где } \quad b \in B, \quad k_{b} \in \mathbb{Z} .
$$


Так как $u=\sum_{b \in B} z_{b} b$, то

$$
u^{T} x=\sum_{b \in B} z_{b} b^{T} x=\sum_{b \in B} z_{b} k_{b}=k_{u} \in \mathbb{Z}
$$

Поэтому точка $x$ лежит в пересечении $\bigcap_{u \in U} H_{k_{u}}(u)$ гиперплоскостей семейств $\mathscr{H}(u)$ для всех $u \in U$. Отсюда вытекает, что множество пересечений всех гиперплоскостей семейств $\mathscr{H}(u)$ для всех $u \in U$ есть точечная решетка $L(U)$, а сами гиперплоскости разбивают все пространство на $n$-мерные многогранники, называемые ячейками.

Очевидно, что начало координат есть точка $0=\bigcap_{u \in U} H_{0}(u)$ решетки $L(U)$. Множество ячеек, имеющих 0 в качестве вершины, называется звездой $\mathrm{St}_{0}$ ячеек в точке $0 \in L(U)$. Каждая ячейка $P \in \mathrm{St}_{0}$ определяется следующим образом:

$$
P=\left\{x \in \mathbb{R}^{n}: 0 \leqslant u^{T} x \leqslant 1 \text { для } u \in U^{+},-1 \leqslant u^{T} x \leqslant 0 \text { для } u \in U^{-}\right\},
$$

где $U^{+} \cup U^{-}=U$ есть некоторое разбиение унимодулярного семейства $U$. Очевидно, что замена $u \in U$ на $-u$ не нарушает унимодулярности семейства. Положив

$$
U^{\prime}=U^{+} \cup\left\{-u: u \in U^{-}\right\}
$$

можно записать многогранник $P$ в виде

$$
P=\left\{x \in \mathbb{R}^{n}: 0 \leqslant u^{T} x \leqslant 1 \text { для всех } u \in U^{\prime}\right\} .
$$

Заметим, что вершины многогранника $P$ суть точки решетки $L(U)$. Поэтому каждое ребро многогранника $P$ имеет в качестве вершин точки $L(U)$. Так как $P$ есть $n$-мерный многогранник, то множество его ребер, имеющих 0 вершиной, содержит множество

$$
E=\left\{e_{i}: 1 \leqslant i \leqslant n\right\}
$$

линейно независимых ребер. Очевидно, что $u^{T} e_{i} \in\{0,1\}$ для любого $u \in U^{\prime}$ и любого $i$. Пусть

$$
E^{*}=\left\{e_{i}^{*}: 1 \leqslant i \leqslant n\right\}
$$

есть базис, двойственный $E$, т.е.

$$
e_{i}^{T} e_{i}^{*}=1, \quad e_{i}^{T} e_{j}^{*}=0, \quad \text { где } \quad 1 \leqslant i, j \leqslant n, \quad, j \neq i .
$$

Теорема 1. Векторы унимодулярной системы $U^{\prime}$ в базисе $E^{*}$ имеют $(0,1)$-координаты.

ДоказАтельство. Пусть $u=\sum_{i=1}^{n} x_{i} e_{i}^{*}$ есть разложение вектора $u \in U^{\prime}$ по векторам базиса $E^{*}$. Тогда для $e_{j} \in E$ имеем

$$
e_{j}^{T} u=\sum_{i=1}^{n} x_{i}\left(e_{j}^{T} e_{i}^{*}\right)=x_{j} .
$$

Но мы видели, что $e_{j}^{T} u \in\{0,1\}$. Утверждение доказано.

Из теоремы 1 вытекает, что для каждого класса эквивалентности $n$-мерных унимодулярных систем существует система подмножеств $n$-множества, характеристические векторы которых образуют унимодулярную систему этого класса. 


\section{СПИСОК ЦИТИРОВАННОЙ ЛИТЕРАТУРЫ}

[1] А. Схрейвер, Теория линейного и иелочисленного программирования. т. 2, Мир, М., 1991. [2] V. Danilov, G. Koshevoy, K. Murota, Math. Social Sci., 41:3 (2001), 251-273. [3] V. I. Danilov, G. A. Koshevoy, Adv. Math., 189:2 (2004), 301-324. [4] R. M. Erdahl, S.S. Ryshkov, European J. Combin., 15:5 (1994), 459-481. [5] P. D. Seymour, J. Combin. Theory Ser. B, 28:3 (1980), 305-359. [6] M. Dutour Sikirić, V. Grishukhin, European J. Combin., 30:4 (2009), 853-865. [7] V. Danilov, V. Grishukhin, European J. Combin., 20:6 (1999), 507-526. [8] G. B. Faulkner, D. H. Younger, Discrete Math., 7:1-2 (1974), 67-74.

\section{В. П. Гришухин}

Центральный экономико-математический институт РАН

E-mail: grishuhn@cemi.rssi.ru

В. И. Данилов

Центральный экономико-математический институт РАН

\section{Г. А. Кошевой}

Центральный экономико-математический институт РАН 